\title{
INDUSTRIA 4.0: ¿CÓMO AFECTA LA DIGITALIZACIÓN AL SISTEMA DE PROTECCIÓN SOCIAL?
}

Industry 4.0: How does digitalization affect the social protection system?

ENEA Ispizua Dorna*

Investigadora doctora de Derecho del Trabajo y de la Seguridad Social.

Universidad del País Vasco/Euskal Herriko Unibertsitatea

\section{RESUMEN}

Se analiza el concepto de la industria 4.0 y el impacto de la cuarta revolución, digitalización y robotización en el mercado de trabajo y especialmente sobre la viabilidad y sostenibilidad financiera del sistema actual de protección social. A tal fin, se analizarán las alternativas o propuestas que plantean algunos autores que van desde la creación de un impuesto específico para los robots, pasando por la cotización social de los robots, hasta la implantación de una renta básica universal.

Palabras clave: digitalización, robotización, seguridad social, renta básica universal, industria 4.0.

\section{ABSTRACT}

We analyze the concept of the industry 4.0 and the impact of the fourth revolution, digitization and robotization have had in the labour market, specifically in the viability and financial sustainability of the current social protection system. To that end, the alternatives or proposals raised by some authors go from the creation of a specific tax for robots, through the social quotation of robots, to the introduction of a universal basic income.

Keywords: digitization, robotization, social security, universal basic income, 4.0 industry.

* Correspondencia a: Enea Ispizua Dorna. Facultad de Derecho. Sección de Bizkaia. Barrio Sarriena s/n. Leioa - enea.ispizua@ ehu.eus - https://orcid.org/0000-0002-7531-8166

Cómo citar: Ispizua Dorna, Enea. (2018). «Industria 4.0: ¿cómo afecta la digitalización al sistema de protección social?»; Lan Harremanak, 40, 12 -30. (https://doi.org/10.1387/lan-harremanak.20325).

Recibido: 31 octubre, 2018; aceptado: 3 diciembre, 2018.

ISSN 1575-7048 - eISSN 2444-5819 / (c) 2018 UPV/EHU

(c) Esta obra está bajo una licencia

Creative Commons Atribución 4.0 Internacional 


\section{SUMARIO}

1. Introducción.-2. ¿¿n qué consiste la industria 4.0?-3. El impacto de la digitalización en el sistema de protección social.—4. La renta básica universal, ¿posible solución?-5. Conclusiones.-6. Bibliografía.

\section{Introducción}

En los últimos años, el entorno laboral está siendo afectado por nuevas formas de organización y retos. La industria comenzó con la primera revolución que fue caracterizada por las grandes empresas y la aparición de las máquinas. Después, con la segunda revolución apareció la producción en cadena y en la tercera revolución, predominaron las nuevas tecnologías de la información y comunicación (TIC). En último lugar, estamos actualmente ante la llegada de la cuarta revolución, donde prima la digitalización de los procesos productivos en las empresas, y en este sentido, aparecen las nuevas formas de organización.

La industria 4.0 tiene ambas caras de la moneda, es decir, cuenta con algunas ventajas y desventajas. Se puede destacar entre las primeras la mayor producción de las empresas, mayores niveles de calidad de los productos y mayor conectividad entre las personas gracias a internet. Por el contrario, entre sus inconvenientes destacan las dificultades de adaptación de las pequeñas y medianas empresas (PYMEs) ante estas nuevas formas de organización, la falta de personal cualificado para poder hacer frente a las nuevas tecnologías, la obsolescencia tecnológica y la pérdida de puestos de trabajo tradicionales. Indudablemente, en esta industria 4.0, los robots sustituyen a los seres humanos en algunos puestos de trabajo y con ello, desaparecen las cotizaciones sociales que aportaban los trabajadores al sistema de la Seguridad Social.

La finalidad de este artículo, por tanto, es analizar el impacto de la digitalización y la robotización en el sistema de protección social. Para esto, en primer lugar, estudiaremos qué es la industria 4.0, para después centrarnos en el análisis del impacto de la digitalización en el sistema de protección social, especialmente en la viabilidad y sostenibilidad del sistema de Seguridad Social actual. Posteriormente, examinaremos la renta básica universal como posible solución a esta situación y finalmente, explicaremos las conclusiones alcanzadas en esta materia. 


\section{2. ¿En qué consiste la industria 4.0?}

Después de varias revoluciones industriales en el siglo xxI, ha llegado la cuarta revolución industrial que incorpora las tecnologías digitales a la industria manufacturera y al sector de servicios (Belstratén, 2018:6-7). Con esta última revolución, el mundo está conociendo nuevas transformaciones tecnológicas como son la digitalización y la inteligencia artificial (Nieto, 2017:1).

En la mitad del siglo XVIII y en las primeras décadas del siglo XIX comenzó la primera revolución industrial en Inglaterra, expandiéndose posteriormente de manera rápida a otros países. En esta revolución predominaban las grandes empresas, se aprovechaban las fuentes de energía como el vapor de agua y surgieron las primeras máquinas.

A mediados del siglo XIx, llegó la segunda revolución industrial y aparecieron nuevas energías como la electricidad y el petróleo y la sociedad empezó a comunicarse a través del telégrafo, teléfono, radio y televisión. Asimismo, en el ámbito laboral se produjeron ciertas modificaciones en la forma de organización, dado que el trabajo se realiza a través de la automatización y especialización. Con esta nueva organización, se desarrollaron las líneas de montaje y la producción en masa y el trabajo en cadena. Algunos autores confirman que la segunda revolución industrial fue un fortalecimiento y perfeccionamiento de la primera revolución industrial.

En la segunda mitad del siglo xx comenzó la tercera revolución industrial y sus pilares según Rifkin son los siguientes: esta revolución industrial se basa en la transición hacia la energía renovable, en la aparición de las energías renovables, en el uso de la tecnología de Internet y en la transición hacia los vehículos de motor eléctrico (Rifkin, 2011:1458). En esta revolución predominan las nuevas tecnologías de la información y la comunicación (TIC), la descentralización de la producción y la utilización de nuevas fuentes de energía, especialmente las renovables.

La cuarta revolución industrial o la denominada «Industria Digital» no significa la prolongación de la tercera, dado que la velocidad en los que se dan los cambios no se puede comparar con ninguna anterior revolución. Esta revolución se ha entendido como la aplicación de los sistemas automatizados, esto es, de los robots con el foco puesto en los procesos productivos. Con ello, se consigue crear redes de producción digitales que permiten aumentar la producción y utilizar los recursos de manera más eficiente. Esta era se caracteriza por el internet de las cosas, a través del cual se consigue mejorar el mantenimiento predictivo y prevenir los fallos en los equipos antes de que ocurran; otra característica es la conectividad, esto es, la conexión de miles de millones de personas mediante internet ha dado lugar al comercio online, a las redes sociales y aplicaciones móviles entre otros; y, por último, la automatización que consiste en que las máqui- 
nas pasan de la actividad programada a trabajar de manera autónoma y flexible (Berger, 2016:12-13). Si además a esto le unimos los avances tecnológicos emergentes como son la inteligencia artificial, la robótica, vehículos autónomos, impresoras 3D, nanotecnología... vemos que los avances son ilimitados (Campero, 2016:3-11). El mundo está conociendo a la vez unas transformaciones tecnológicas de nuevo tipo mediante la digitalización y de la inteligencia artificial.

¿Dónde se encuentra el origen del concepto «Industria 4.0»? La industria 4.0 es una iniciativa estratégica impulsada por el gobierno alemán donde recoge algunas recomendaciones para hacer frente a los retos que plantea el objetivo europeo «Horizonte 2020» (Castresana, 2016:12). El gobierno alemán lo describió como "una organización de los procesos de producción basada en la tecnología y en dispositivos que se comunican entre ellos de forma autónoma a lo largo de la cadena de valor» (Blanco et al., 2017:151). De esta manera se consigue aumentar la optimización del control de los procesos de trabajo y de las cadenas de suministro. Según un informe elaborado por el Ministerio de Industria, Energía y Turismo, la industria 4.0 surge como "una nueva revolución industrial que consiste en incorporar las nuevas tecnologías (cloud, sistemas ciber-físicos, sensórica, entre muchas otras) a la industria. Se trata de un nuevo camino industrial que ya están recorriendo varios paises». En este mismo informe se destaca el desafío que supone la industria 4.0 para Espańa, pero también resalta la posibilidad u oportunidad que puede ofrecer para mejorar su posición competitiva (Ministerio de industria y turismo, 2014:26).

En la figura de la página siguiente podemos observar la evolución de la industria que hemos comentado y en qué consiste cada etapa de manera resumida (Ministerio de industria y turismo, 2014:26)

Las principales características de la industria 4.0, podemos clasificarlas de la siguiente manera (Alzaga et al., 2016) (IBM, Centro español de Logística: 8):

- Se trata de un modelo flexible, esto es, la manera de producir es personalizada. De esta manera, se conecta a los proveedores, clientes y empleados en todo el proceso de elaboración del producto.

- Se basa en la re-configurabilidad, de manera que existe una capacidad de adaptación de forma rápida y económica ante los cambios en el producto.

- Existe la digitalización de los procesos, debido a que hay una conectividad e integridad entre las diferentes fases del proceso productivo.

- Se caracteriza por la «Smartización», lo que supone aprender de las experiencias previas.

- Los empleados trabajan con herramientas digitales como son, por ejemplo, los robots colaborativos y sistemas de inteligencia artificial y esto hace que aumente la productividad y la seguridad. 


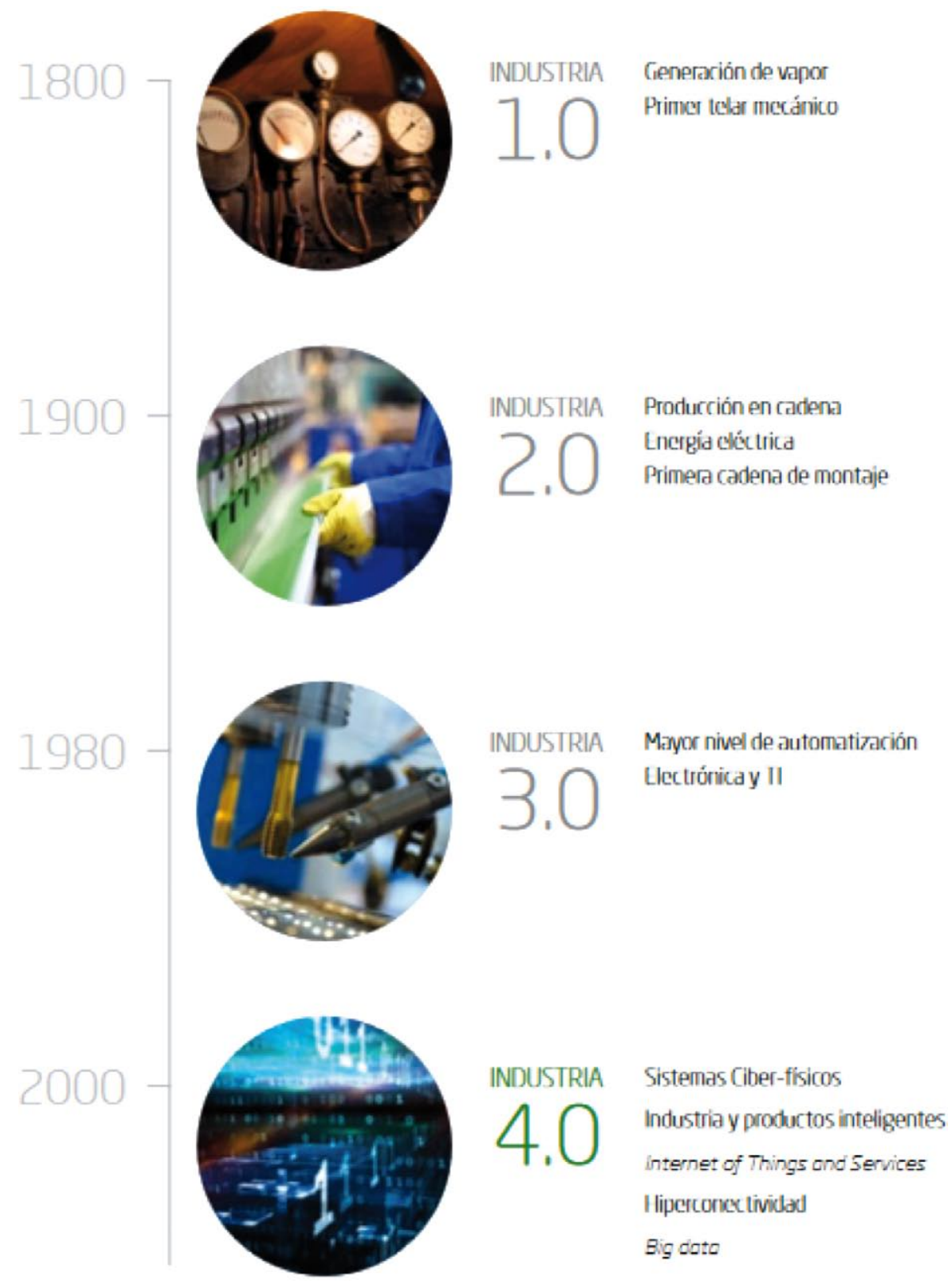

Evolución de la industria

Fuente: Informe Industria conectada 4.0. http://www6.mityc.es/IndustriaConectada40/informeindustria-conectada40.pdf 
Además de las características mencionadas, en la industria 4.0 existen sistemas ciber-físicos, como son Big Data y Cloud Computing y robots colaborativos de simulación, realidad aumentada, visión artificial...Estos nuevos mecanismos no fracasarán si se dan ciertos requisitos como son, los cambios en la organización del trabajo para adaptarse a los nuevos modelos de negocio y si se les da la formación necesaria a los trabajadores y estos últimos muestran disponibilidad en investigación y desarrollo (Blanco et al., 2017:154). No obstante, estos últimos requisitos no serán suficientes si no se acompañan con una regulación específica que desempeñe el legislador. El legislador deberá tener en cuenta los cambios que se produzcan a la hora de modificar y desarrollar nuevas normas, y también deberá apoyar a las pequeñas y medianas empresas (PYMEs) ante estos cambios organizativos, dado que éstas se encuentran con diversos problemas: muchas veces desconocen los avances tecnológicos existentes, o aun teniendo la información se encuentran con la insuficiente financiación para efectuar la transformación digital. El legislador ya ha comenzado a desempeñar sus funciones de legislar ante estas nuevas circunstancias, por ejemplo, con la entrada en vigor el 25 de mayo de 2018 del Reglamento (UE) 2016/679 del Parlamento Europeo y del Consejo de 27 de abril de 2016 relativo a la protección de las personas físicas en lo que respecta al tratamiento de los datos personales y a la libre circulación de estos datos. No obstante, tiene muchos aspectos que debe regular, como puede ser el sistema formativo de la formación profesional como universitaria respecto a los nuevos perfiles que se crearán relacionadas con la industria 4.0, la seguridad y salud de los trabajadores, la protección social... (Blanco et al., 2017:154).

Se puede constatar que la industria 4.0 ha generado grandes incertidumbres, que algunos aspectos se pueden trasladar en beneficiosos, pero otros pueden resultar perjudiciales. En cuanto a los primeros, podemos destacar las ventajas económicas que se pueden generar y la reducción de las tasas de siniestralidad. En este sentido, la transformación digital puede anticipar los fallos en la producción, puede reducir el absentismo laboral debido a la reducción de los índices de frecuencia y la gravedad de los accidentes de trabajo y también pueden aumentar el valor del producto que ponen en el mercado las empresas (Buisán et al., 2017:90). En esta nueva era parece carecer de sentido la deslocalización, debido a que en revoluciones anteriores era muy común que las empresas desarrollasen su fase de producción en países donde el coste de la mano de obra era inferior. No obstante, con esta nueva revolución se recuperan todos los procesos de la cadena de valor y en consecuencia, se generan más puestos de trabajo donde se encuentra la empresa (CCOO Industria, 2017:2). Respecto a las desventajas o perjuicios que puede causar la industria 4.0 se pueden destacar, en primer lugar, la destrucción de empleo, debido a que muchas máquinas interactúan sin necesidad de la intervención del ser humano (CCOO Industria, 2017:2). Esta digitalización también producirá un impacto en la seguridad y salud en el trabajo, 
creándose nuevos riesgos especialmente los psicosociales. Además, se plantean dudas respecto a qué ocurrirá con las pensiones, ya que el aumento de los robots puede traer consigo la pérdida de empleos y en efecto, la pérdida del número de cotizantes de la Seguridad Social. Trataremos esta materia en los siguientes apartados.

\section{El impacto de la digitalización en el sistema de protección social}

La industria 4.0 se presenta como una amenaza de algunos empleos y profesiones tradicionales. Los cambios en el mundo del trabajo son muy rápidos debido a que las nuevas tecnologías van avanzando constantemente y de manera rápida. Con la entrada de la digitalización y robotización se han generado diversas dudas respecto al futuro del empleo y, sobre todo, respecto a la viabilidad de los sistemas recaudatorios, tanto tributarios como de Seguridad Social. No podemos únicamente tener en cuenta los efectos que tienen estos mecanismos nuevos a corto plazo, sino también hay que prestar atención a las consecuencias a largo plazo como es el caso de la sostenibilidad del sistema público de las pensiones (Sanchéz-Urán et al., 2015:32). Además, en esos efectos directamente vinculados con las nuevas tecnologías no sólo podemos considerar a los robots, ya que éstos llevan tiempo siendo incorporados, sino también debemos tener en cuenta los efectos de Internet, de la impresión 3D, la inteligencia artificial... Si estas nuevas tecnologías sustituyen puestos de trabajo, se pierden los recursos financieros que asumen los trabajadores respecto a las cotizaciones que realizan. Además, si se destruyen puestos de trabajo, un efecto directo de ello será el aumento indudable de las prestaciones por desempleo y, en consecuencia, se producirán mayores dificultades a la hora de financiar las futuras pensiones de jubilación (Cuatrecasas, Instituto de Estrategia Legal en RRHH, 2018: 332-333).

Estos nuevos desafíos ya están siendo debatidos en la UE, debido a las preocupaciones que han surgido al respecto. Así, en un dictamen del Comité Económico y Social Europeo se planteaba que «el fuerte incremento de las modalidades atipicas de empleo ocasionado por la digitalización implica que una parte cada vez mayor de la mano de obra dejará de contribuir y de beneficiarse de los sistemas establecidos de seguridad social, como los subsidios de desempleo, la sanidad pública y los seguros de pensiones...». También se resalta en el mismo, que este impacto negativo se observa tanto en el empleo como en los ingresos de los regímenes fiscales y de los sistemas de bienestar social, los cuales principalmente se financian a través de los impuestos y cotizaciones de los empresarios y trabajadores. Por tanto, estamos ante unos sistemas que dependen de unos niveles elevados de los empleos tradicionales. Ante esta situación, en algunos Estados miembros, los interlocutores sociales y el gobierno han comenzado a debatir respecto al tema (Dictamen del Comité Económico y Social Europeo, 2016). Asimismo, la Re- 
solución del Parlamento Europeo, de 16 de febrero de 2017, realizó una serie de recomendaciones destinadas a la Comisión sobre normas de Derecho civil sobre robótica. Esta resolución en primer lugar, destacó que, aunque el uso de los robots no acarree automáticamente la sustitución de los puestos de trabajo, es probable que los empleos menos cualificados en sectores donde predomina la mano de obra sean más vulnerables a la automatización. Ante este panorama, a través de esta resolución propone una serie de recomendaciones en distintos ámbitos, pero nosotros nos centraremos en las recomendaciones relacionadas con la educación y el empleo (Resolución del Parlamento Europeo, de 16 de febrero de 2017, con recomendaciones destinadas a la Comisión sobre normas de Derecho Civil sobre robótica, 2015) (López, 2017):

En primer lugar, insta a la Comisión que preste apoyo en el desarrollo de las competencias digitales en todos los grupos de edad y en todas las categorías profesionales con el objetivo de mejorar la adaptación de la oferta y demanda en el mercado de trabajo. Asimismo, destaca que la formación o conocimientos adquiridos en los centros escolares no es suficiente, debido a que la velocidad de las nuevas tecnologías es muy rápida y, por tanto, el aprendizaje es continuo a lo largo de toda la vida.

En segundo lugar, constata el aspecto positivo de la robótica en relación a la salud y seguridad en el trabajo, dado que pueden mejorar las condiciones de trabajo, asumiendo los robots las tareas perjudiciales y peligrosas que desempeñan los trabajadores.

En tercer lugar, el Parlamento Europeo pide a la Comisión que analice los posibles efectos y consecuencias en la viabilidad de los sistemas de Seguridad Social de los Estados miembros, sobre todo ante el reto del envejecimiento de la población. Sin embargo, únicamente se ha realizado este mero pronunciamiento, sin realizar ningún desarrollo respecto al debate sobre el pago de impuestos por parte de los robots o su contribución en las cotizaciones sociales. Por lo tanto, esta recomendación puede resultar pobre teniendo en cuenta el gran interés y el debate suscitado en esta materia. Asimismo, llama la atención que las recomendaciones previas que se propusieron a través del informe de propuesta del día 27 de enero de 2017, añadían la necesidad de emprender «un debate integrador sobre los nuevos modelos de empleo y sobre la sostenibilidad de nuestros sistemas tributarios y sociales tomando como base unos ingresos suficientes, incluida la posible introducción de una renta básica minima» (Recomendación 44) (Resolución del Parlamento Europeo, de 16 de febrero de 2017, con recomendaciones destinadas a la Comisión sobre normas de Derecho Civil sobre robótica, 2015) que no fueron recogidos en el texto final. Incluso de forma más específica, la Comisión de Empleo y Asuntos Sociales entre sus sugerencias destacaba que "debido al desarrollo y la utilización de robots colaborativos inteligentes y la inteligencia artificial, el diferencial entre la creación y la pérdida de em- 
pleo podría repercutir en la sostenibilidad financiera de los regimenes de seguridad social, los regímenes de pensiones y los sistemas de seguro de desempleo de los Estados miembros..." (Informe con recomendaciones destinadas a la Comisión sobre normas de Derecho Civil sobre robótica).

A nivel europeo, es de destacar igualmente, la propuesta innovadora que plantea la eurodiputada Mady Delvaux ante el peligro de la viabilidad de los sistemas de seguridad social en consecuencia del futuro de la robotización en el empleo. La eurodiputada cree que si no se toman medidas al respecto podrán aparecer mayores brechas en la distribución de la riqueza y el poder. En este sentido, su propuesta se basa en que los Estados miembros exijan a las empresas que se encuentran en su Estado a que informen acerca de en qué medida y proporción los robots contribuyen a sus resultados económicos. Así, posteriormente, se podrán determinar los correspondientes impuestos y cotizaciones al sistema de la Seguridad Social. No obstante, resulta difícil pensar que las empresas desempeñen esta función de manera voluntaria, dado que podría acabar perjudicándoles (Sierra, 2017:133-159).

En el ámbito nacional, al igual que en el resto de Estados miembros, estos cambios afectan de manera directa a nuestro sistema de Seguridad Social, debido a que se basa en un sistema de reparto y de prestación definida. Esta forma de reparto de las pensiones se organiza en una base de aporte obligatorio que es realizado por los trabajadores que se encuentran en activo para atender las pensiones y jubilaciones de los trabajadores inactivos. Por lo tanto, se rige por el principio de solidaridad, es decir, la generación que cotiza financia la pensión de la generación jubilada y a su vez, la primera será financiada por la siguiente generación. Este último principio se aprecia en las prestaciones por desempleo. En este sentido, la financiación de la Seguridad Social podemos decir que se encuentra condicionada principalmente por la situación del empleo y, por ende, con la era de la digitalización y la introducción de los robots, este sistema puede tambalear y deberán buscarse otras alternativas para paliar el descenso de ingresos del sistema de Seguridad Social. Además de la incorporación de los robots y la digitalización, no debemos olvidar el envejecimiento de la población como un reto fundamental de este sistema de reparto. Ante esta panorámica, algunas de las opciones que los autores proponen son los siguientes: la primera alternativa consiste en establecer la obligación de que las empresas que sustituyan puestos de trabajo ocupados por personas por robots realicen aportaciones específicas al sistema de Seguridad Social y la segunda alternativa se basa en establecer un tributo específico a las empresas por la sustitución de trabajadores por robots. En cualquiera de las dos opciones, el legislador cuando vaya a realizar las modificaciones legislativas oportunas, deberá ser cauteloso para que la solución no impacte negativamente en la productividad y la competitividad de las empresas, y tampoco en la creación de nuevos puestos de trabajo relacionados con estas nuevas formas de organización (Cuatrecasas, Instituto de Estrategia Legal 
en RRHH, 2018:333). A estas dos opciones debemos añadirle otra que consiste en la solución de la renta básica universal que trataremos de abordar en el siguiente apartado. No obstante, ¿las dos opciones predominantes entre los autores resultan viables?

La primera alternativa, es decir, la obligatoriedad de que las empresas que sustituyan puestos de trabajo ocupados por personas por robots realicen aportaciones específicas al sistema de Seguridad Social, genera muchísimas dudas que deberían ser aclaradas por el legislador en caso de optar por esta opción como vía de solución a la viabilidad de las pensiones. Muchos autores demuestran que el papel que la administración debe desempeñar en estos procesos es muy importante para minimizar los riesgos. Sin embargo, se plantean diversas cuestiones como: ¿por qué robots deberían las empresas cotizar? Esto es, ¿por todos los robots o únicamente por los robots inteligentes autónomos? Asimismo, ¿quién es el sujeto activo de esa obligación de cotizar? ¿cuál es la cuantía que debería cotizar al sistema público de Seguridad Social? ¿durante cuánto tiempo debe mantenerse esa cotización por parte de la empresa? ¿qué ocurre en caso de que el empresario sustituya un robot por otro? ¿Se mantiene dicha obligación de cotizar en este último supuesto?

Para poder resolver estas preguntas, es fundamental que el legislador defina qué es un robot y no parece resultar sencillo. Asimismo, en la regulación actual de la Seguridad Social ¿dónde encajarían los robots? El artículo 7.2. del Real Decreto 2064/1995, de 22 de diciembre, por el que se aprobó el Régimen General sobre Cotización y Liquidación de otros Derechos de la Seguridad Social establece quiénes son los sujetos de la cotización. Respecto a los sujetos activos determina que "están sujetas a la obligación de cotizar a la Seguridad Social las personas físicas o jurídicas, en los términos y condiciones que se determinen en el presente Reglamento para cada uno de los diferentes regimenes que integran el sistema de la Seguridad Social...» (Real Decreto 2064/1995, de 22 de diciembre, por el que se aprueba el Reglamento General sobre cotización y liquidación de otros derechos de la Seguridad Social). Como podemos observar en este precepto, son las personas físicas o jurídicas los que deben cotizar al sistema. Parece entonces que los robots no encajan en esta disposición. Además, si analizamos el artículo 1 del Estatuto de los Trabajadores, donde se recogen cuáles son las notas definitorias de una relación laboral y nos centramos en la nota de la voluntariedad podríamos constatar que los robots tampoco son considerados como trabajadores. No obstante, si se quiere implantar esta alternativa, algunos autores encuentran la solución a este precepto mencionado en que el sujeto obligado de la cotización sea la empresa que ha optado por la robotización. Así, la empresa encuadraría en la disposición del Real Decreto, al considerarse persona jurídica. Y aún más, el empresario respetaría el principio de solidaridad recogido en el artículo 2.1 de la Ley General de la Seguridad Social, donde se determina que «el sistema de la Seguridad Social, configurado por la acción protectora en sus modalidades contribu- 
tiva y no contributiva, se fundamenta en los principios de universalidad, unidad, solidaridad e igualdad» (Real Decreto Legislativo 8/2015, de 30 de octubre, por el que se aprueba el texto refundido de la Ley General de la Seguridad Social). Este principio de solidaridad del empresario tiene su fundamentación en la compensación por la sustitución del empleo humano y la consiguiente reducción de los ingresos en el sistema de la Seguridad Social, debido a que el empresario al optar por los robots consigue mayores beneficios (Cuatrecasas, Instituto de Estrategia Legal en RRHH, 2018: 335). Una vez determinado el sujeto activo quién sería en estos casos, se plantea la cuestión de si el empresario debe cotizar por todas las máquinas o robots o por algunos determinados. Esta duda planteó el Parlamento Europeo en el anexo a la propuesta de Resolución de recomendaciones destinadas a la Comisión sobre normas de Derecho Civil sobre robótica. Dicho anexo promovía una definición europea común de robots autónomos inteligentes que incluyesen las siguientes características (Anexo del informe con recomendaciones destinadas a la Comisión sobre normas de Derecho Civil sobre robótica, cita textual):

— La capacidad de adquirir autonomía mediante sensores y/o mediante el intercambio de datos con su entorno (interconectividad) y el análisis de dichos datos.

— La capacidad de aprender a través de la experiencia y la interacción.

- La forma del soporte físico del robot.

- La capacidad de adaptar su comportamiento y acciones al entorno.

Por lo tanto, con este planteamiento que realiza el Parlamento Europeo únicamente cotizaría la empresa por los robots que hayan sustituido a personas en el trabajo y que reúnan las características mencionadas. Parece que se trata de una solución limitada. En consecuencia, y teniendo en cuenta dicha limitación, algunos autores abogan por una aplicación más extensa que consistiría en incluir a todos aquellos robots o máquinas que supongan una reducción del número de trabajadores, limitándolo a un porcentaje. Esto es, el legislador debería concretar un porcentaje y si el empresario rebasa dicha cantidad entonces existiría la posibilidad de exigirle el pago de cotizaciones al sistema de Seguridad Social (Cuatrecasas, Instituto de Estrategia Legal en RRHH, 2018:336). No obstante, creo que esta alternativa tiene principalmente un problema: ¿cómo va a saber el legislador o el administrador si una empresa concreta alcanza el límite establecido? Dudo mucho que el empresario vaya a proporcionarle dicha información de manera voluntaria si posteriormente puede resultarle perjudicial. Por lo tanto, el legislador debería establecer a las empresas la obligatoriedad de conceder dicha información.

Dentro de la obligación de cotización que tendría el empresario, ¿a qué cantidad nos referimos? Es otra de las cuestiones debatidas y muchos autores están de acuerdo en que la cotización podría ser inferior a la vinculada con 
un trabajador, básicamente porque los robots no causarían derecho a prestaciones del sistema de Seguridad Social. Esto es, las cotizaciones de estos robots estarían basados en el principio de solidaridad del sistema de Seguridad Social y no estarían vinculadas a contingencias concretas, como pueden ser el desempleo y la jubilación. Relacionado con este tema, también se plantea la duda de cuándo se termina la obligación de cotizar por los robots. Se han concedido distintas soluciones a esta pregunta: por un lado, algunos consideran que la cotización finaliza cuando el robot pasa a estar inactivo; por otro lado, hay quienes opinan que termina dicha cotización cuando el robot deja de desempeñar la actividad; y finalmente, otros opinan que finaliza la cotización cuando se sustituye por otro robot (Cuatrecasas, Instituto de Estrategia Legal en RRHH, 2018:336).

Obviamente, todas estas cuestiones que han sido planteadas y son debatidas, deberán ser clarificarlas por los poderes públicos, y el legislador tendrá la labor de modificar las normas actuales o crear unas nuevas para adaptarlas a estos cambios organizativos. En especial, sería fundamental modificar la Ley General de la Seguridad Social e incluir a estos robots en las disposiciones correspondientes a la obligación de cotizar que actualmente no se encuentran.

La segunda disyuntiva, esto es, establecer una imposición fiscal específica a las empresas por la sustitución de trabajador por robots, tampoco se desvincula de las complicaciones y dudas que genera. La idea de un impuesto específico al trabajo robótico que sería pagado por las empresas que utilicen no ha sido aprobado en ninguna etapa histórica. En la revolución industrial se desarrollaron todo tipo de máquinas y apareció la automatización de la producción y, en consecuencia, desaparecieron muchos puestos de trabajo que desempeñaban los obreros. Y hasta la actualidad, han pasado décadas donde se han ido adoptando nuevas tecnologías productivas, aumentando la productividad y los beneficios. Esto podría haber generado la creación de una tasación específica, pero no se ha planteado hasta ahora. El razonamiento de este impuesto específico podría basarse en que el empresario al emplear robots pasa a tener más beneficios, debido a que la calidad del producto que ofrece es mayor junto al aumento de su productividad y además disminuye la cantidad de nóminas que tenía anteriormente (Dans, 2017). Esta alternativa ya se mencionaba en el apartado K del Informe con recomendaciones destinadas la Comisión sobre normas de derecho civil sobre robótica que elaboró el Parlamento Europeo: "... deberá estudiarse la posibilidad de someter a impuesto el trabajo ejecutado por robots o exigir un gravamen por el uso y mantenimiento de cada robot, a fin de mantener la cohesión social y la prosperidad» (Informe con recomendaciones destinadas a la Comisión sobre normas de Derecho Civil sobre robótica). Posteriormente en la Resolución se replanteó esta alternativa y finalmente, no se recogió por considerarse que podría afectar directamente al avance de la innovación. Asimismo, debemos unir a este problema otro que es fundamental, con motivo de distribución de com- 
petencias de los Estados miembros. Esto es, en la Unión Europea cada Estado miembro dispone de la competencia de regulación en el ámbito fiscal y, en consecuencia, pueden producirse desigualdades y asimetrías entre los países. Un efecto inmediato de esta circunstancia puede ser que algunas empresas se trasladen a otros países, consiguiendo con esa huida evadir o disminuir los impuestos (Dans, 2017).

De la misma manera que comentábamos en las cotizaciones de la seguridad social, en esta solución también es necesaria la definición legal de qué es un robot para posteriormente poder atribuirle efectos tributarios. Asimismo, el sujeto activo no sería el robot por no tener capacidad financiera, ni poseer capital ni liquidez, por lo que le correspondería en este caso también al empresario el pago de dicho impuesto. Un elevado número de autores cree que es más conveniente imponer el impuesto sobre las ganancias que obtiene el empresario por el uso de dichos robots que sobre los mismos robots. En este sentido un eurodiputado del PSOE propone que a las empresas cuyos beneficios tengan relación directa y probada con la actividad robótica se les grave con un porcentaje más alto sobre sus beneficios (Villarino, 2017).

Hay que tener en cuenta que cualquiera de las dos opciones mencionadas puede suponer un perjuicio para la innovación y el desarrollo tecnológico y, en consecuencia, también para el empleo a medio y largo plazo. El legislador deberá tener en cuenta estos inconvenientes a la hora de determinar cuál es la solución adecuada para enfrentarnos al problema de la viabilidad y sostenibilidad del sistema actual de Seguridad Social.

\section{La renta básica universal, ¿posible solución?}

Durante los últimos años y como efecto directo de la crisis económica, el envejecimiento de la población y la implantación de los robots y la digitalización, la viabilidad y sostenibilidad de las pensiones ha sido muy debatida y cuestionada. Ante esta situación la argumentación de que el Estado de Bienestar ha de reformarse ha aumentado considerablemente y los autores han prestado una creciente atención a la innovadora propuesta de la creación de una Renta Básica universal o de ciudadanía. Esta propuesta consiste en "el pago por parte de la administración de una prestación monetaria a todos los ciudadanos, sin tener en cuenta su renta, su historial laboral o disponibilidad para el empleo, ni la composición de su hogar familiar». En resumen, es una prestación totalmente universal, individual e incondicional, que se cobraría por el mero hecho de existir como miembro de una comunidad política (Noguera, 2014:1). Teniendo en cuenta las características de dicha renta se puede decir que todo ciudadano tendrá garantizado un umbral de ingresos, por lo que las actuales prestaciones asisten- 
ciales y no contributivas carecerían de sentido y quedarían absorbidas por esta renta básica. Como expresa NOGUERA, «ningún beneficiario de prestaciones sociales vería empeorada su prestación con una Renta Básica y es más muchos la verían mejorada» (Noguera, 2014:1). Y reflexiona que la Renta Básica únicamente reemplazaría a las prestaciones monetarias que se encuentren por debajo de la establecida por la Renta Básica. De esta manera, el resto de prestaciones se mantendrían como están actualmente. Es importante analizar qué es lo que significa el concepto Renta Básica Universal. En este sentido, podemos realizar una clasificación de las características (Raventós et al., 2018:13-16):

1. Básica: ‘a qué se refiere? El objetivo de que la renta sea básica supone que debe proporcionar una seguridad económica básica, pero no una seguridad total. En este sentido, la mayoría de los defensores de esta renta creen que debería ser reconocido como un derecho. Por tanto, como derecho no podría ser anulado y además no podría ser incautada por impago de deudas.

2. Universal: se entiende por universal que dicha renta sería pagada a cada residente habitual de una comunidad, provincia o país.

3. Individual: la renta básica se pagaría a cada individuo sin tener en cuenta su estatus marital, familiar o doméstico. En este aspecto, hay autores que defienden pagar una cantidad menor a los niños, que sería gestionado por su madre. Asimismo, algunos proponen pagar suplementos a dicha renta a los pensionistas y discapacitados, para compensar su coste de vida mayor y su pequeña probabilidad de ganar otros ingresos adicionales.

4. Incondicional: el ciudadano no tendría que probar que tiene una renta por debajo de cierta cantidad y tampoco existirían condiciones de gasto, es decir, el ciudadano no tendría que justificar en qué y dónde gasta el dinero. Asimismo, no existirían condiciones de comportamiento, por ejemplo, aceptando determinados empleos, o teniendo que estar dispuestos a hacerlo para poder percibir la renta, como ocurre ahora con la prestación de desempleo.

5. Regular: la renta sería pagada de manera regular y la recomendación habitual es que sea mensual.

Como hemos comentado anteriormente, la robotización y la digitalización hará que desaparezcan los trabajos tradicionales y se crearán nuevos trabajos relacionados con estas nuevas formas de organización. Asimismo, con la automatización y el uso de los robots aumentará la productividad y, por tanto, esos beneficios extraordinarios conseguidos por el aumento de dicha productividad podrían ser destinados a una Renta Básica Universal. Entre los autores que investigan sobre este tipo de renta, existen todo tipo de opiniones. Los que abogan por esta renta destacan sus ventajas (Noguera, 2014:4-9): 
- Con la implantación de esta renta universal vinculada a cada ciudadano, se observa una mejora respecto a las negociaciones de las relaciones laborales, debido a que el ciudadano dispone de un suelo sobre el que podrá apoyarse a la hora de negociar con el empresario. Su libertad de negociar es más amplia, acabando con el miedo y angustia.

— La Renta Básica reemplaza las exenciones y bonificaciones que puede tener el empresario a la Seguridad Social.

— La Renta Básica no estaría gravada por el IRPF.

- Aumentaría la seguridad económica para los ciudadanos.

- La cobertura que alcanzaría la Renta Básica universal abarca a todos los ciudadanos y, por tanto, se evitaría cualquier exclusión.

- Para poder percibir la Renta Básica no sería necesaria su solicitud, sino se otorgaría automáticamente a todo ciudadano.

- La aceptación de un trabajo no supondría la extinción de la renta, y en este sentido, desaparecería el desincentivo al empleo como ocurre actualmente con las prestaciones sociales condicionadas como es el caso de la prestación por desempleo.

- Se reducirían los gastos administrativos, ya que no existiría ningún control ni comprobación en el abono de dicha renta, y entonces su gestión sería más sencilla.

- El Estado abonaría la Renta de oficio, sin que existiese ninguna discrecionalidad al otorgarla por parte de las diferentes administraciones.

- La Renta Básica integraría muchas de las actuales prestaciones asistenciales y así, se evitaría que, en períodos de crisis económica, se colapsasen los servicios asistenciales y sociales.

En cambio, los detractores de la Renta Básica Universal argumentan que la implantación de dicha renta llevaría a que habría mucha menos gente que encontrase un empleo y que los trabajos penosos serían llevados a cabo por extranjeros que no tengan derecho a percibir dicha renta básica, sin que fueran incrementadas las retribuciones de estos trabajos (Piazuelo, 2016). Asimismo, destacan otro problema relacionado con la inmigración y el control de sus fronteras, derivado del efecto llamada que podría producirse por esa redistribución de la riqueza.

En este sentido, también debemos tener en cuenta cuáles podrían ser las estrategias políticas de implantación de dicha renta básica universal. Parece que existen distintas fórmulas, pero la mayoría traen consigo una serie de problemas (Noguera, 2014:11-13).

En primer lugar, podría aplicarse una estrategia de introducción parcial de la Renta Básica respecto a su cuantía e ir aumentándola poco a poco. Sin embargo, ésta demuestra una serie de desventajas, debido a que muchos de los efectos positivos previstos con la implantación de la renta básica universal, no se 
visualizarían. Al tratarse además de una cantidad reducida no podrían ser absorbidos por ésta el resto de prestaciones, sino que deberían ser mantenidas con carácter complementario. Con esto, estaríamos ante una administración y gestión más compleja.

En segundo lugar, se plantea la posibilidad de introducir la renta básica de manera plena, pero por colectivos de población, por ejemplo, a los más desfavorecidos. En este caso, podrían aparecer supuestos de discriminación por las diferencias de cobertura de los distintos colectivos y también supondría aumentar las funciones de la administración en cuanto al control de medios.

La tercera estrategia se basaría en introducir gradualmente la Renta Básica, reformando al mismo tiempo programas que ya existen de garantía de mínimo de rentas. En cuanto a las prestaciones no contributivas se podrían reformar las rentas mínimas de inserción, otras prestaciones existentes en el ámbito de las Comunidades Autónomas, los subsidios de desempleo...

Parece quedar claro que no existe una única fórmula de implantación de la Renta Básica Universal, y en caso de que el legislador alcance un acuerdo para su creación, debería observar los distintos medios para su establecimiento y su viabilidad.

Si contemplamos otros Estados Miembros de la Unión Europea, podemos destacar Finlandia por ser el laboratorio europeo de la renta básica universal. En enero de 2017, este país puso en marcha un proyecto piloto para un grupo limitado de 2000 desempleados que perciben 560 euros mensuales hasta el 31 de diciembre de 2018. Esta cantidad ha sido destinada a desempleados comprendidos entre 25 y 58 años. Los perceptores de la renta continúan recibiéndola cuando encuentran trabajo (Ministerio de Empleo y Seguridad Social, Secretaría general técnica, 2018:105). Sin embargo, a finales de diciembre finalizará el proyecto y no se dará continuidad. El gobierno finlandés prefiere explorar otras medidas menos costosas y que impliquen menos cambios a nivel fiscal.

\section{Conclusiones}

Con las anteriores reflexiones ha quedado claro que la industria 4.0 ha llegado para quedarse en nuestro mercado laboral y las empresas se encontrarán con nuevos retos que afrontar, debido a que la tecnología avanza constantemente. En la industria 4.0 todos los procesos se encuentran interconectados a través de internet y el mercado y si las empresas consiguen adaptarse a estas nuevas formas de organización, podrán mejorar su productividad. Sin embargo, en esta transformación deberán participar también los poderes públicos junto a las 
empresas, ofreciendo programas de formación y además los trabajadores deberán mostrar su disponibilidad para enfrentarse a esos cambios.

Uno de los retos que plantea la industria 4.0 está relacionada con la protección social y en concreto, con la viabilidad de nuestro sistema actual de Seguridad Social. Así lo demuestra la Comisión Europea cuando urge a los Estados miembros a ir adaptando la legislación a un mundo donde las máquinas ganarán protagonismo hasta convertirse en el centro de gravedad del proceso productivo. Insta a que se cree un instrumento legislativo para gobernar la robótica y la inteligencia artificial, teniendo en cuenta también entre ellos el sistema de Seguridad Social.

La robotización y digitalización trae consigo la desaparición de trabajos tradicionales, pero también destacan los autores que aparecerán otros nuevos. Por lo tanto, es muy importante la formación de los trabajadores ante estos nuevos trabajos para competir mejor en el mercado laboral del futuro.

Otra de las consecuencias inmediatas de esta robotización se encuentra en el descenso de las cotizaciones sociales y en efecto, una menor cantidad en la caja del sistema de la Seguridad Social. En consecuencia, como hemos podido observar, han sido varias las alternativas que se han propuesto, entre ellas establecer un gravamen fiscal o el deber de cotizar por los robots. No obstante, estas propuestas pueden generar un impacto negativo: la robotización se implanta en las empresas con el fin de mejorar la productividad y si se establece un gravamen, puede traer consigo una consecuencia negativa que es la desincentivación de la inversión tecnológica, la cual es necesaria para mejorar el tejido productivo. Asimismo, el poner trabas a la robótica puede frenar el desarrollo de las empresas europeas y dar ventaja competitiva a otros países del mundo.

Está claro, por tanto, que el legislador deberá ser cauteloso a la hora de determinar qué alternativa puede aplicar, dado que los robots dan valor añadido a las empresas y además puede suponer la recuperación de industrias que acudieron a otros estados donde la mano de obra era más barata.

Otra alternativa que han propuesto muchos autores se basa en la creación de la renta básica universal con los beneficios extra generados por la robotización. Sin embargo, la aprobación de una renta básica constituiría, un cambio o reforma profunda de algunos de los principales rasgos de las políticas de protección social actuales. 


\section{Bibliografía}

Alzaga, Aitor y Larreina, Jon (2016), “¿Qué es la industria 4.0?», interempresas.net, recuperado de http://www.interempresas.net/Robotica/Articulos/150900-Que-es-laIndustria-40.html (acceso: 30-11-2018).

Berger, Roland (2016), «España 4.0. El reto de la transformación digital de la economía», w5.siemens.com, recuperado de https://w5.siemens.com/spain/web/es/estudio digitalizacion/Documents/Estudio_Digitalizacion_Espana40_Siemens.pdf (acceso: 30-11-2018).

Bestratén Belloví, Manuel, Gavilanes Pérez, Cecilia y Gómez-Cano Alfaro, María (2018), "Revolución 4.0: el futuro está presente», Seguridad y Salud en el trabajo, n. 94

Blanco, Raúl, Fontrodona, Jordi y Poveda, Carmen (2017), «La industria 4.0: el estado de la cuestión", Ministerio de Industria, Comercio y Turismo, recuperado de https://www.mincotur.gob.es/Publicaciones/Publicacionesperiodicas/Economia Industrial/RevistaEconomiaIndustrial/406/BLANCO,\%20FONTRODONA\%20 Y\%20POVEDA.pdf (acceso: 30-11-2018).

Buisán, Mario y ValdÉs, Fernando (2017), «La industria conectada 4.0», Información Comercial Española, ICE: Revista de economía, n. ${ }^{\circ} 898$.

Campero Núñez del Prado, José Carlos (2016), «¿La cuarta revolución industrial en Bolivia?», Friedrich Ebert Stiftung, N.o 1, recuperado de https://library.fes.de/pdffiles/bueros/bolivien/14338.pdf (30-11-2018)

Castresana Sáenz, Carolina (2016), Industria 4.0., Universidad de La Rioja, recuperado de https://biblioteca.unirioja.es/tfe_e/TFE002004.pdf (acceso: 30-11-2018).

CCOO INDUSTRIA (2017), «La digitalización y la industria 4.0, Impacto industrial y laboral», Madrid, recuperado de http://www.industria.ccoo.es/4290fc51a3697f785ba14fc e86528e10000060.pdf (acceso: 30-11-2018)

Cuatrecasas, Instituto de Estrategía legal en RRHH (2018), Robótica y su impacto en los Recursos Humanos y en el Marco Regulatorio de las Relaciones Laborales, Wolters Kluwer España.

DANs, Enrique (2017), «Robots e impuestos: no tan sencillo», recuperado de https://www. enriquedans.com/2017/02/robots-e-impuestos-no-tan-sencillo.html (acceso: 30-112018).

IBM, Centro español de Logística, «La gestión de la cadena de suministro en la era de la Industria 4.0».

López Cumbre, Lourdes (2017), «Robots con derechos y obligaciones civiles... y laborales», Análisis GA\&P.

Ministerio de Empleo y Seguridad Social, Secretaría General Técnica (2018), «Finlandia. La RENTA Básica Universal Versus el «modelo de activación», Revista de Actualidad Internacional, n. ${ }^{\circ} 219$.

Nieto, Joaquín (2017), «El futuro del trabajo que queremos y el derecho del trabajo, IusLabor 3/2017.

Noguera, José Antonio (2014), «La renta básica universal: razones y estrategias», Fundación Centro de Estudios Andaluces, Junta de Andalucía, recuperado de https://www.centrodeestudiosandaluces.es/datos/factoriaideas/policypaper_5.pdf (acceso: 30-11-2018).

Piazuelo plou, Antonio, "Renta básica universal. ¿Un estado de bienestar para el siglo XxI?», Cortes de Aragón, recuperado de http://www.sinpermiso.info/printpdf/ 
textos/prologo-al-libro-renta-basica-universal-un-estado-de-bienestar-para-el-siglo-xxi (acceso: 30-11-2018).

Raventós, Daniel y Casassas, David (2018), La Renta Básica. Un derecho para todos y para siempre, Pasado \& presente, Barcelona.

Rifkin, Jeremy (2011), La tercera revolución industrial, trad. de Albino Santos Mosquera, España, Paidós.

Sanchez-Urán Azaña, M. ${ }^{\text {a }}$ Yolanda. y Grau Ruiz, M. a Amparo (2015), «El impacto de la robótica, en especial la robótica inclusiva, en el trabajo: aspectos jurídico-laborales y fiscales", Inclusive Robotics for a better Society. Recuperado de http://inbots.eu/wpcontent/uploads/2018/08/publications/robotica-derecho-del-trabajo-derecho-fiscal-final-mayo2018.pdf (acceso: 30-11-2018),

Sierra Benítez, Esperanza Macarena (2017), «La protección social de los trabajadores ante el desafío del nuevo trabajo a distancia, del trabajo digital y la robótica», Revista de Derecho de la Seguridad Social, n. ${ }^{\circ} 11$, Ed. Laborum, Murcia.

Villarino, Ángel (2017), «Robots que cotizan y pagan multas de tráfico: Europa imagina leyes para máquinas», recuperado de https:/www.elconfidencial.com/ tecnologia/2017-02-18/robots-cotizan-pensiones-futuro-multas-trafico-legislacion_ $1334077 /(30-11-2018)$. 